\title{
Associação entre o estado de saúde autorreferido de adultos e a área de localização do domicílio: uma análise de regressão logística ordinal usando a PNAD 2008
}

\author{
Association between self-reported state of health among adults \\ and the location of the home: ordinal logistic regression \\ analysis using PNAD 2008
}

José Rodrigo de Moraes ${ }^{1}$

Jessica Pronestino de Lima Moreira ${ }^{2}$

Ronir Raggio Luiz ${ }^{2}$

${ }^{1}$ Departamento de Estatística, Instituto de Matemática e Estatística, Universidade Federal Fluminense. R. Mário

Santos Braga s/n, Centro. 24020-140 Niterói Rio de Janeiro.

jrodrigo78@gmail.com

${ }^{2}$ Instituto de Estudos em

Saúde Coletiva,

Universidade Federal do

Rio de Janeiro

\begin{abstract}
The urban environment influences human health and behavior, and a clearer understanding of the health determinants of populations living in cities is needed. Using data from the $\mathrm{Na}$ tional Household Sampling Survey (PNAD) 2008, with ordinal logistic models incorporating the sampling plan, this study assessed the association between the location of the home (urban or rural) and the self-reported state of health of the adult Brazilian population, taking into account a set of individual and environmental factors inside and outside the home. The results indicated that after allowing for individual and environmental factors, the association between the location of the home and the self-reported state of health is changing (going from $O R=1.51$ to $O R=0.96$ ) and losing its statistical significance ( $p$-value $=0.208$ ). However, statistically significant interactions were observed between the location of the home and the following variables: sex, color/race, self-reported morbidity, possession of basic goods and percentage of households with adequate living conditions. Key words Self-reported state of health, Location of the home, Ordinal logistic regression model
\end{abstract}

Resumo O ambiente urbano influencia a saúde e os comportamentos humanos, sendo necessário um melhor entendimento dos determinantes da saúde das populações que vivem nas cidades. A partir dos dados da Pesquisa Nacional por Amostra de Domicílios (PNAD) 2008, usando modelos logísticos ordinais incorporando o plano amostral, o presente artigo avalia a associação entre a área de localização do domicílio (urbana e rural) e o estado de saúde autorreferido da população adulta brasileira, controlando para um conjunto de fatores individuais e do ambiente intradomiciliar e extradomiciliar. Os resultados indicam que ao controlar por fatores individuais e ambientais, a associação entre a área de localização do domicílio e o estado de saúde autorreferido dos adultos se modifica (passando de $O R=1,51$ para $O R=0,96)$ e perde a sua significância estatística (p-valor $=0,208)$. Entretanto, foram observadas interações estatisticamente significantes entre a área de localização do domicílio e as seguintes variáveis: sexo, cor/raça, morbidade autorreferida, posse de bens básicos e percentual de domicílios adequados quanto a qualidade de moradia.

Palavras-chave Estado de saúde autorreferido, Área de localização do domicílio, Modelo de regressão logística ordinal 


\section{Introdução}

O ambiente urbano influencia a saúde e os comportamentos humanos, apontando para a necessidade de melhor entendimento dos determinantes da saúde das populações que vivem nas cidades $^{1}$. No Brasil, houve um aumento na taxa de urbanização, passando de $81,2 \%$ em 2000 para $83,8 \%$ em 2008, que em termos absolutos significa mais de 159 milhões de pessoas vivendo nas cidades em $2008^{2}$.

Com relação aos múltiplos efeitos da urbanização na saúde da população ainda há divergências entre estudos. Alguns, sobre a relação entre o processo de urbanização e a saúde da população, apontam como fatores que proporcionam uma melhor saúde dos indivíduos em áreas urbanas, comparativamente a áreas rurais, um maior nível de renda, educação e informação, que lhes proporcionam um melhor acesso a uma alimentação de qualidade ${ }^{3,4}$ e um maior acesso de serviços de saúde ${ }^{5}$. Por outro lado, outros estudos apontam que o processo de urbanização, sobretudo quando não planejado, pode trazer uma série de riscos a saúde da população como resultado de residências impróprias, aglomeração humana, poluição ambiental, água para consumo insuficiente ou contaminada, instalações sanitárias inadequadas, esgoto não-tratado, estresses associados com pobreza e desemprego ${ }^{6,7}$.

Segundo Caiaffa et al. ${ }^{1}$, independente do efeito positivo ou negativo da vida nas cidades contemporâneas (meio urbano), o conceito de saúde deve incorporar o cotidiano dos indivíduos vivendo nelas, não ignorando as relações de interdependência que existem entre eles e o meio físico, social e político onde vivem e se inserem. Os resultados em saúde parecem, pois depender de quem se é e do lugar onde se vive. Apreende-se, portanto que o estudo dos determinantes de saúde não pode se limitar a fatores individuais, devendo ser considerados também fatores ambientais para explicar as diferenças nos níveis de saúde de populações humanas. A importância da consideração de indicadores relativos à qualidade das moradias está no fato delas representarem conceitos de privação real, ou seja, indicam que os indivíduos estão efetivamente privados ${ }^{4}$.

Do ponto de vista da prevenção das doenças e da promoção da qualidade de vida e das condições de saúde no país é fundamental identificar fatores, sobretudo os passíveis de intervenção (modificáveis) que influenciam os níveis de saúde de populações humanas, com o objetivo de reduzir as iniqüidades em saúde. Entretanto, o estabelecimento desta relação não é simples, não só por causa da multiplicidade de fatores envolvidos e pelas inter-relações existentes entre eles $(. . .)^{4}$, mas, sobretudo pelas limitações provenientes do tipo de desenho de estudo epidemiológico adotado e da disponibilidade e qualidade das informações nos níveis geográficos requeridos.

$\mathrm{Na}$ análise das diferenças nos níveis de saúde de populações humanas considerando variáveis individuais e contextuais, têm sido frequentemente utilizados, como proxy das avaliações objetivas de saúde individual, indicadores de autopercepção do estado global de saúde, por ser de fácil mensuração, confiável e válido ${ }^{8-10}$, além de permitir comparações internacionais ${ }^{11}$.

Vários autores utilizam o indicador de autopercepação do estado de saúde em seus estudos ${ }^{4,12-}$ ${ }^{14}$, entretanto diferem quanto à forma de tratar $\mathrm{o}$ indicador. Por exemplo, Dachs e Santos ${ }^{13}$, com base nos dados da PNAD 2003, usaram modelos de regressão logística binária para relacionar a autopercepção do estado de saúde dicotomizada em ruim/muito ruim ou não com um conjunto de determinantes individuais. Já Nogueira ${ }^{4}$ usando modelos de regressão logística ordinal relacionou a autopercepção do estado de saúde com um conjunto de determinantes individuais e contextuais na Área Metropolitana de Lisboa.

No presente artigo são analisadas as diferenças no estado de saúde autorreferido dos adultos usando dados mais recentes da PNAD utilizada por Dachs e Santos ${ }^{13}$ e o mesmo tipo de modelo adotado por Nogueira ${ }^{4}$, mas incorporando as informações do plano amostral. Considerando a população adulta residente em domicílios particulares permanentes que informou o seu próprio estado de saúde, este artigo tem como objetivo avaliar a associação entre a área de localização do domicílio (urbana e rural) e o estado de saúde autorreferido, controlando para um conjunto de variáveis demográficas, socioeconômicas, comportamentais e de saúde que retratam características individuais e do ambiente intradomiciliar e extradomiciliar.

\section{Material e métodos}

\section{Pesquisa Nacional por Amostra de Domicílios}

A Pesquisa Nacional por Amostra de Domicílios (PNAD) é de abrangência nacional, realizada pelo Instituto Brasileiro de Geografia e Estatística (IBGE), que coletou informações de uma 
amostra probabilística de 150.591 domicílios e 391.868 pessoas. Tem como finalidade produzir informações básicas para o estudo do desenvolvimento socioeconômico do País. Sua amostra foi planejada para obter estimativas representativas para o Brasil, Grandes Regiões, Unidades da Federação e nove Regiões Metropolitanas (Belém, Fortaleza, Recife, Salvador, Belo Horizonte, Rio de Janeiro, São Paulo, Curitiba e Porto Alegre). Com relação ao desenho amostral, a PNAD é um estudo seccional que combina estratificação, conglomeração em dois ou três estágios, com probabilidades desiguais de seleção que resultam em pesos amostrais distintos para os elementos da amostra. Maiores informações sobre o planejamento amostral podem ser obtidos em Silva et al. ${ }^{15}$ e IBGE 2 .

\section{População de estudo}

A população-alvo deste estudo compreende os indivíduos adultos (20 anos ou mais de idade) residentes em domicílios particulares permanentes que informaram o seu próprio estado de saúde, o que corresponde a uma seleção de 162.213 registros na amostra da PNAD. Foram consideradas na análise apenas as pessoas que informaram seu próprio estado global de saúde, por entender que informações prestadas por terceiros (outras pessoas moradoras do domicílio, ou ainda outras pessoas não moradoras do domicílio) aumentaria a chance de ocorrer viés de informação ou classificação do desfecho de saúde utilzado neste trabalho. Do total de adultos residentes em domicílios particulares permanentes, 37,2\% tiveram seu estado de saúde informado por terceiros.

\section{Variáveis de estudo}

A partir das cinco alternativas de resposta do quesito de autopercepção do estado global de saúde obteve-se um desfecho ordinal com três níveis: muito bom/bom, regular, ruim/muito ruim.

A área de localização do domicílio classificada em urbana ou rural, de acordo com a legislação vigente na ocasião do Censo Demográfico 2000, é a variável de exposição principal, cuja associação com o estado de saúde autorreferido se deseja avaliar em nível nacional. A área urbana foi definida como agregação de três categorias da variável original situação censitária disponível no banco de dados da PNAD 2008: 1) Cidade ou vila, área urbanizada; 2) Cidade ou vila, área não-urbanizada; e 3 ) Área urbana isolada. A área rural, por sua vez, foi definida como junção de cinco categorias da variável situação censitária: 1) Aglomerado rural de extensão urbana; 2) Aglomerado rural, isolado, povoado; 3) Aglomerado rural, isolado, núcleo; 4) Aglomerado rural, isolado, outros aglomerados; e 5) Zona rural exclusive aglomerado rural.

Como variáveis de controle foi utilizado um conjunto de dezoito variáveis demográficas, socioeconômicas, comportamentais e de saúde que retratam características individuais e do ambiente intradomiciliar e extradomiciliar: sexo, faixa etária, cor/raça, escolaridade, situação de ocupação, atividade física, fumo, morbidade autorreferida, mobilidade física, posse de plano de saúde, consulta médica nos últimos 12 meses, domicílio cadastrado no Programa de Saúde da Família (PSF), qualidade de moradia, posse de bens básicos no domicílio, condição de ocupação do domicílio, renda domiciliar mensal per capita, região de residência e proporção de domicílios considerados adequados no setor (vizinhança) quanto aos serviços sociais básicos (água, esgoto, lixo e luz), as densidades habitacionais e aos padrões construtivos das moradias (material usado nas paredes e telhado). As variáveis de controle que foram obtidas através da agregação de variáveis disponíveis no banco de dados da PNAD 2008 são descritas abaixo.

A variável qualidade de moradia representa uma característica do ambiente intradomiciliar, construída através da combinação de sete variáveis binárias que indicam adequação ou inadequação de três dimensões distintas (qualidade da construção, infra-estrutura de serviços sociais e densidade domiciliar) de acordo com os critérios definidos na publicação da Pesquisa sobre Padrões de Vida 1996-1997'16. As categorias da variável qualidade da moradia são: adequado para os domicílios considerados adequados em todos os indicadores das três dimensões e inadequado - para os domicílios considerados inadequados em pelo menos um dos indicadores de alguma das dimensões. A partir da variável qualidade de moradia, foi obtida uma outra variável que retrata uma característica do ambiente extradomiciliar, através do cálculo do percentual de domicílios existentes no setor considerados adequados quanto à qualidade da construção, à infra-estrutura de serviços sociais (água, esgoto, lixo e luz) e à densidade habitacional.

A posse de bens básicos no domicílio é uma outra variável do ambiente intradomiciliar, com duas categorias que informam se no domicílio existem todos os quatro bens básicos (fogão, geladeira, televisão e rádio) ou se não existe pelo menos um desses bens. 
A variável mobilidade física foi construída a partir do agrupamento das respostas (não consegue, tem grande dificuldade, tem pequena dificuldade ou não tem dificuldade) de oito quesitos de mobilidade física levantados na PNAD 2008, resultando em quatro níveis que retratam os diferentes estados de mobilidade física dos indivíduos ou diferentes graus de limitação na realização de determinadas atividades básicas do cotidiano: muita limitação, limitação, pouca limitação e sem limitação. Os indivíduos são classificados como tendo muita limitação quando não conseguem ou têm grande dificuldade para realizar as atividades básicas de alimentação e higiene, ou não conseguem realizar pelo menos uma das demais atividades. Como tendo limitação quando os indivíduos têm pequena ou nenhuma dificuldade nas atividades de alimentação e higiene, mas possui grande dificuldade em pelo menos uma das outras atividades. Como tendo pouca limitação, os indivíduos que não têm pequena ou nenhuma dificuldade, mas têm pequena dificuldade em pelo menos uma das outras atividades. Finalmente, foram classificados como sem limitação, os indivíduos que não apresentaram nenhuma dificuldade em todas as atividades.

A variável morbidade autorreferida foi construída a partir das respostas (sim ou não) de doze quesitos que investigam a presença de doze doenças crônicas. As categorias da variável morbidade autorreferida informam se o indivíduo não possui nenhuma destas 12 doenças crônicas ou se possui pelo menos uma destas doenças.

\section{Análise Estatística}

Utilizando o software SPSS for Windows, versão 17.0 (SPSS Inc., Chicago, Estados Unidos), foram ajustados modelos logísticos ordinais para o estado de saúde autorreferido, considerando os pesos amostrais e as informações estruturais do plano amostral (estratos e unidades primárias de amostragem) da PNAD 2008. Nos ajustes foram usados pesos amostrais corrigidos definidos pela razão entre os pesos naturais do desenho e a sua média aritmética, para manter o uso do tamanho de amostra na realização das inferências realizadas ${ }^{17}$. Neste artigo foi utilizado o modelo logístico ordinal, também chamado de modelo de chances proporcionais, cuja forma funcional está representada em Abreu et al ${ }^{18}$, pois é o mais utilizado por investigadores sociais na área de saúde e está implementado na maioria dos pacotes estatísticos que possuem rotinas para a análise de dados amostrais complexos (SPSS, STATA, SAS, etc.), como os dados da PNAD.
Além dos efeitos principais das dezoito variáveis de controle, também foram testados os efeitos de interação das variáveis selecionadas com a área de localização do domicílio.

\section{Resultados}

Quase 70\% da população adulta brasileira avaliou o seu estado global de saúde como bom ou muito bom. Apenas 5,6\% dos adultos avaliaram o seu estado de saúde como ruim ou muito ruim, enquanto os demais $(26,0 \%)$ reportaram um estado de saúde regular (Gráfico 1).

A Tabela 1 apresenta os resultados dos modelos logísticos ordinais univariados relacionando todas as variáveis consideradas com a autopercepção do estado de saúde dos adultos, além de suas distribuições de freqüências simples e conjuntas (com a autopercepção do estado de saúde).

Dos adultos que informaram seu próprio estado de saúde, 83,3\% residem em domicílios localizados na área urbana. Com relação à distribuição da autopercepção do estado de saúde segundo as categorias das variáveis consideradas, pode-se destacar também que a proporção de adultos com estado de saúde autorreferido bom ou muito bom é maior entre os residentes na área urbana $(70,0 \%)$, na região Sudeste (72,5\%), em domicílios com os quatro bens básicos $(70,1 \%)$ e adequados com relação à densidade e à infra-estrutura física e de serviços sociais $(71,8 \%)$. Pode-se destacar ainda que esta proporção também é maior entre indivíduos que nunca fumaram $(73,1 \%)$, que praticaram atividade física $(80,5 \%)$, sem nenhuma doença crônica $(86,6 \%)$, com plano de saúde $(78,0 \%)$ e que não consultaram médico nos últimos 12 meses $(81,3 \%)$ (Tabela 1).

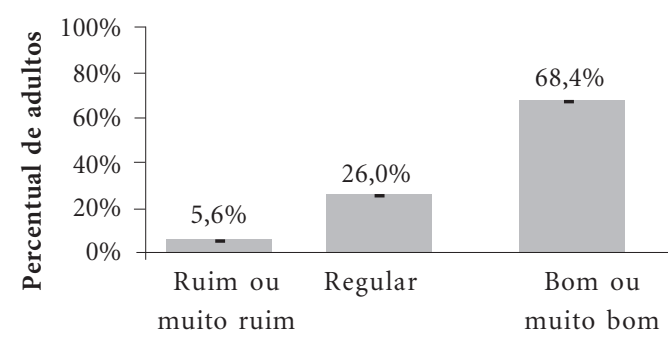

Estado de saúde autorreferido

Gráfico 1. Distribuição de adultos segundo o estado de saúde autorreferido - Brasil 
Tabela 1. Associações de variáveis demográficas, socioeconômicas, comportamentais e de saúde com o estado de saúde autorreferido - Brasil.

\begin{tabular}{|c|c|c|c|c|c|c|c|}
\hline \multirow[t]{2}{*}{ Variáveis } & \multirow{2}{*}{$\begin{array}{l}\text { Distribuição } \\
\text { percentual de } \\
\text { adultos }\end{array}$} & \multicolumn{3}{|c|}{$\begin{array}{l}\text { Distribuição percentual de } \\
\text { adultos segundo o estado } \\
\text { de saúde autorreferido }\end{array}$} & \multicolumn{3}{|c|}{$\begin{array}{c}\text { Modelos logísticos } \\
\text { ordinais univariados }\end{array}$} \\
\hline & & $\begin{array}{l}\text { Ruim ou } \\
\text { muito ruim }\end{array}$ & Regular & Total & $\begin{array}{l}\text { Razão de } \\
\text { chance }\end{array}$ & IC $95 \%$ & p-valor \\
\hline \multicolumn{8}{|l|}{$\begin{array}{l}\text { Área de localização do } \\
\text { domicílio }\end{array}$} \\
\hline Urbano & 83,3 & 5,3 & 24,7 & 100 & 1,51 & {$[1,43 ; 1,59]$} & $<0,001$ \\
\hline Rural & 16,7 & 7,3 & 32,3 & 100 & 1 & - & - \\
\hline \multicolumn{8}{|l|}{ Região de residência } \\
\hline Norte & 7,4 & 6,3 & 28,8 & 100 & 0,82 & {$[0,75 ; 0,89]$} & $<0,001$ \\
\hline Nordeste & 27,7 & 7,0 & 30,6 & 100 & 0,73 & {$\left[\begin{array}{ll}0,69 & 0,77\end{array}\right]$} & $<0,001$ \\
\hline Sudeste & 42,0 & 4,7 & 22,8 & 100 & 1,16 & {$[1,09 ; 1,23]$} & $<0,001$ \\
\hline Sul & 15,4 & 5,7 & 25,0 & 100 & 0,99 & {$[0,92 ; 1,06]$} & 0,701 \\
\hline Centro-oeste & 7,4 & 4,9 & 25,7 & 100 & - & - & - \\
\hline \multicolumn{8}{|l|}{ Sexo } \\
\hline Masculino & 38,4 & 5,3 & 23,2 & 100 & 1,26 & {$[1,23 ; 1,29]$} & $<0,001$ \\
\hline Feminino & 61,6 & 5,9 & 27,7 & 100 & 1 & - & - \\
\hline \multicolumn{8}{|l|}{ Faixa etária } \\
\hline 20 a 29 anos & 22,3 & 1,4 & 13,0 & 100 & 7,26 & {$[6,93 ; 7,60]$} & $<0,001$ \\
\hline 30 a 39 anos & 22,1 & 2,4 & 18,6 & 100 & 4,59 & {$[4,41 ; 4,79]$} & $<0,001$ \\
\hline 40 a 49 anos & 20,6 & 5,0 & 26,5 & 100 & 2,64 & {$[2,54 ; 2,74]$} & $<0,001$ \\
\hline 50 a 59 anos & 16,0 & 8,6 & 34,7 & 100 & 1,58 & {$[1,51 ; 1,64]$} & $<0,001$ \\
\hline 60 anos ou + & 18,9 & 12,6 & 41,9 & 100 & 1 & - & - \\
\hline \multicolumn{8}{|l|}{ Cor/raça } \\
\hline Branca & 49,0 & 4,9 & 23,2 & 100 & 1,38 & {$[1,34 ; 1,42]$} & $<0,001$ \\
\hline Não branca & 51,0 & 6,3 & 28,7 & 100 & 1 & - & - \\
\hline \multicolumn{8}{|l|}{ Escolaridade } \\
\hline $\begin{array}{l}\text { Sem instrução ou menos } \\
\text { de } 1 \text { ano }\end{array}$ & 13,0 & 14,9 & 40,0 & 100 & 0,11 & {$[0,10 ; 0,11]$} & $<0,001$ \\
\hline 1 a 7 anos & 36,2 & 7,4 & 33,8 & 100 & 0,20 & {$[0,18 ; 0,21]$} & $<0,001$ \\
\hline 8 a 14 anos & 42,1 & 2,3 & 17,9 & 100 & 0,54 & {$[0,51 ; 0,58]$} & $<0,001$ \\
\hline 15 anos ou + & 8,5 & 1,2 & 10,8 & 100 & 1 & - & - \\
\hline Sem declaração & 0,2 & 5,0 & 25,5 & 100 & 0,31 & {$[0,23 ; 0,42]$} & $<0,001$ \\
\hline \multicolumn{8}{|l|}{$\begin{array}{l}\text { Renda domiciliar mensal } \\
\text { per capita }\end{array}$} \\
\hline $\begin{array}{l}\text { Sem renda ou até } 1 \\
\text { salário mínimo }\end{array}$ & 51,0 & 7,4 & 30,3 & 100 & 0,26 & {$[0,24 ; 0,28]$} & $<0,001$ \\
\hline $\begin{array}{l}\text { Mais de } 1 \text { a } 5 \text { salários } \\
\text { mínimos }\end{array}$ & 40,7 & 4,1 & 22,5 & 100 & 0,44 & {$[0,41 ; 0,48]$} & $<0,001$ \\
\hline $\begin{array}{l}\text { Mais de } 5 \text { salários } \\
\text { mínimos }\end{array}$ & 5,4 & 1,5 & 12,4 & 100 & 1 & - & - \\
\hline Sem declaração & 2,8 & 4,8 & 23,6 & 100 & 0,40 & {$[0,36 ; 0,45]$} & $<0,001$ \\
\hline \multicolumn{8}{|l|}{ Situação de ocupação } \\
\hline Ocupada & 62,2 & 3,5 & 22,1 & 100 & 2,12 & {$[2,06 ; 2,17]$} & $<0,001$ \\
\hline Não ocupada & 37,8 & 9,2 & 32,3 & 100 & 1 & - & - \\
\hline \multicolumn{8}{|l|}{ Atividade física } \\
\hline Pratica & 25,2 & 2,0 & 17,5 & 100 & 2,15 & {$[2,08 ; 2,23]$} & $<0,001$ \\
\hline Não pratica & 72,3 & 5,7 & 28,4 & 100 & 1 & - & - \\
\hline Sem declaração & 2,5 & 40,8 & 41 & 100 & 0,09 & {$[0,09 ; 0,10]$} & $<0,001$ \\
\hline \multicolumn{8}{|l|}{ Fumo } \\
\hline Fumante & 15,4 & 7,2 & 28,0 & 100 & 0,67 & {$[0,64 ; 0,69]$} & $<0,001$ \\
\hline Ex-fumante & 16,7 & 8,9 & 33,8 & 100 & 0,49 & {$[0,48 ; 0,51]$} & $<0,001$ \\
\hline Nunca fumou & 50,9 & 4,1 & 22,9 & 100 & 1 & - & - \\
\hline Sem declaração & 17,0 & 5,7 & 25,8 & 100 & 0,79 & {$[0,77 ; 0,82]$} & $<0,001$ \\
\hline
\end{tabular}


Tabela 1. continuação

\begin{tabular}{|c|c|c|c|c|c|c|c|}
\hline \multirow[t]{2}{*}{ Variáveis } & \multirow{2}{*}{$\begin{array}{l}\text { Distribuição } \\
\text { percentual de } \\
\text { adultos }\end{array}$} & \multicolumn{3}{|c|}{$\begin{array}{l}\text { Distribuição percentual de } \\
\text { adultos segundo o estado } \\
\text { de saúde autorreferido }\end{array}$} & \multicolumn{3}{|c|}{$\begin{array}{l}\text { Modelos logísticos } \\
\text { ordinais univariados }\end{array}$} \\
\hline & & $\begin{array}{l}\text { Ruim ou } \\
\text { muito ruim }\end{array}$ & Regular & Total & $\begin{array}{l}\text { Razão de } \\
\text { chance }\end{array}$ & IC $95 \%$ & p-valor ${ }^{*}$ \\
\hline \multicolumn{8}{|l|}{ Morbidade autorreferida } \\
\hline $\begin{array}{l}\text { Pelo menos uma doença } \\
\text { crônica }\end{array}$ & 46,5 & 10,9 & 41,7 & 100 & 0,14 & {$[0,13 ; 0,14]$} & $<0,001$ \\
\hline $\begin{array}{l}\text { Nenhuma doença } \\
\text { crônica }\end{array}$ & 53,5 & 1,1 & 12,3 & 100 & 1 & - & - \\
\hline \multicolumn{8}{|l|}{ Mobilidade física } \\
\hline Muita limitação & 8,1 & 29,0 & 48,2 & 100 & 0,04 & {$[0,03 ; 0,04]$} & $<0,001$ \\
\hline Limitação & 12,9 & 16,0 & 53,2 & 100 & 0,08 & {$[0,07 ; 0,08]$} & $<0,001$ \\
\hline Pouca limitação & 15,6 & 4,3 & 39,9 & 100 & 0,23 & {$[0,22 ; 0,24]$} & $<0,001$ \\
\hline Sem limitação & 63,4 & 0,9 & 14,1 & 100 & 1 & - & - \\
\hline \multicolumn{8}{|l|}{ Posse de plano de saúde } \\
\hline Sim & 27,2 & 3,0 & 19,0 & 100 & 1,94 & {$[1,87 ; 2,01]$} & $<0,001$ \\
\hline Não & 72,8 & 6,6 & 28,6 & 100 & 1 & - & - \\
\hline \multicolumn{8}{|l|}{ Consulta médica } \\
\hline Sim & 73,9 & 6,9 & 29,3 & 100 & 0,40 & {$[0,39 ; 0,41]$} & $<0,001$ \\
\hline Não & 26,1 & 2,0 & 16,7 & 100 & 1 & - & - \\
\hline \multicolumn{8}{|l|}{ PSF } \\
\hline Sim & 49,4 & 6,6 & 29,5 & 100 & 0,66 & {$[0,64 ; 0,69]$} & $<0,001$ \\
\hline Não & 50,6 & 4,7 & 22,5 & 100 & 1 & - & - \\
\hline \multicolumn{8}{|l|}{$\begin{array}{l}\text { Condição de ocupação do } \\
\text { domicílio }\end{array}$} \\
\hline Próprio & 76,1 & 6,1 & 27,2 & 100 & 1,17 & {$[0,97 ; 1,41]$} & 0,098 \\
\hline Alugado & 15,4 & 3,6 & 20,2 & 100 & 1,88 & {$[1,55 ; 2,28]$} & $<0,001$ \\
\hline Cedido & 8,0 & 5,1 & 25,7 & 100 & 1,32 & {$[1,09 ; 1,60]$} & 0,005 \\
\hline Outra & 0,5 & 8,0 & 28,6 & 100 & 1 & - & - \\
\hline \multicolumn{8}{|l|}{ Posse de bens básicos } \\
\hline $\begin{array}{l}\text { Tem todos os quatro } \\
\text { bens básicos }\end{array}$ & 82,6 & 4,9 & 24,9 & 100 & 1,59 & {$[1,53 ; 1,65]$} & $<0,001$ \\
\hline $\begin{array}{l}\text { Não tem pelo menos um } \\
\text { bem básico }\end{array}$ & 17,4 & 9,0 & 30,8 & 100 & 1 & - & - \\
\hline \multicolumn{8}{|l|}{ Qualidade da moradia } \\
\hline Adequado & 54,1 & 4,8 & 23,4 & 100 & 1,41 & {$[1,36 ; 1,46]$} & $<0,001$ \\
\hline Não adequado & 45,9 & 6,6 & 29,1 & 100 & 1 & - & - \\
\hline$\%$ domicílios adequados & & & & & 2,00 & {$[1,90 ; 2,11]$} & $<0,001$ \\
\hline
\end{tabular}

^Teste Wald

Analisando os resultados dos modelos logísticos ordinais univariados (Tabela 1), observase que todas as características demográficas, socioeconômicas, comportamentais e de saúde (individuais ou dos ambientes intra e extradomiciliar) estão estatisticamente relacionadas com a chance do adulto autorreferir um melhor estado de saúde ao nível de significância de 5\%. Na análise univariada (análise bruta) observou-se que a chance do adulto apresentar um melhor estado de saúde autorreferido na área urbana é 51\% maior que na área rural $(\mathrm{OR}=1,51$; $\mathrm{IC} 95 \%=$ $[1,43 ; 1,59])$.

A Tabela 2 apresenta os resultados do ajuste dos modelos ordinais multivariados para avaliar a associação entre a área de localização do domicílio e a autopercepção do estado de saúde dos adultos, controlando simultaneamente pelas variáveis ambientais e individuais previamente apresentadas, fixando o nível de significância em 5\%.

Considerando primeiramente todos os efeitos principais das variáveis no ajuste do modelo 
Tabela 2. Modelos logísticos ordinais (multivariados) explicativos do estado de saúde autorreferido de adultos - Brasil.

\begin{tabular}{|c|c|c|c|c|c|c|}
\hline \multirow[t]{2}{*}{ Variáveis } & \multicolumn{3}{|c|}{$\begin{array}{l}\text { Modelo multivariado } 1: \\
\text { inclui os efeitos principais } \\
\text { significativos }\end{array}$} & \multicolumn{3}{|c|}{$\begin{array}{l}\text { Modelo multivariado 2: } \\
\text { inclui os efeitos principais e } \\
\text { de interação significativos }\end{array}$} \\
\hline & $\begin{array}{l}\text { Razão de } \\
\text { chance }\end{array}$ & IC $95 \%$ & p-valor ${ }^{*}$ & $\begin{array}{l}\text { Razão de } \\
\text { chance }\end{array}$ & IC $95 \%$ & p-valor ${ }^{*}$ \\
\hline \multicolumn{7}{|l|}{ Área de localização do domicílio } \\
\hline Urbano & 0,96 & {$[0,90 ; 1,02]$} & 0,208 & 0,93 & {$[0,83 ; 1,05]$} & 0,226 \\
\hline Rural & 1 & - & - & 1 & - & - \\
\hline \multicolumn{7}{|l|}{ Região de residência } \\
\hline Norte & 0,75 & {$[0,68 ; 0,83]$} & $<0,001$ & 0,75 & {$[0,68 ; 0,83]$} & $<0,001$ \\
\hline Nordeste & 0,78 & {$[0,74 ; 0,84]$} & $<0,001$ & 0,78 & {$[0,73 ; 0,83]$} & $<0,001$ \\
\hline Sudeste & 1,10 & {$[1,04 ; 1,17]$} & 0,002 & 1,10 & {$[1,03 ; 1,17]$} & 0,003 \\
\hline Sul & 0,99 & {$[0,91 ; 1,07]$} & 0,722 & 0,99 & {$[0,92 ; 1,07]$} & 0,819 \\
\hline Centro-oeste & 1 & - & - & 1 & - & - \\
\hline \multicolumn{7}{|l|}{ Sexo } \\
\hline Masculino & 0,85 & {$[0,83 ; 0,88]$} & $<0,001$ & 0,96 & {$[0,90 ; 1,02]$} & 0,187 \\
\hline Feminino & 1 & - & - & 1 & - & - \\
\hline \multicolumn{7}{|l|}{ Faixa etária } \\
\hline 20 a 29 anos & 1,41 & {$[1,33 ; 1,49]$} & $<0,001$ & 1,42 & {$[1,34 ; 1,50]$} & $<0,001$ \\
\hline 30 a 39 anos & 1,19 & {$[1,14 ; 1,25]$} & $<0,001$ & 1,20 & {$[1,14 ; 1,26]$} & $<0,001$ \\
\hline 40 a 49 anos & 0,96 & {$[0,92 ; 1,00]$} & 0,067 & 0,96 & {$[0,92 ; 1,01]$} & 0,101 \\
\hline 50 a 59 anos & 0,83 & {$[0,80 ; 0,87]$} & $<0,001$ & 0,84 & {$[0,80 ; 0,87]$} & $<0,001$ \\
\hline 60 anos ou + & 1 & - & - & 1 & - & - \\
\hline \multicolumn{7}{|l|}{ Cor/raça } \\
\hline Branca & 1,16 & {$[1,12 ; 1,20]$} & $<0,001$ & 1,02 & {$[0,95 ; 1,10]$} & 0,618 \\
\hline Não branca & 1 & & & 1 & & - \\
\hline \multicolumn{7}{|l|}{ Escolaridade } \\
\hline Sem instrução ou menos de 1 ano & 0,46 & {$[0,42 ; 0,50]$} & $<0,001$ & 0,46 & {$[0,42 ; 0,50]$} & $<0,001$ \\
\hline 1 a 7 anos & 0,47 & {$[0,44 ; 0,51]$} & $<0,001$ & 0,48 & {$[0,45 ; 0,52]$} & $<0,001$ \\
\hline 8 a 14 anos & 0,69 & {$[0,65 ; 0,74]$} & $<0,001$ & 0,70 & {$[0,65 ; 0,75]$} & $<0,001$ \\
\hline 15 anos ou + & & & - & 1 & & - \\
\hline Sem declaração & 0,46 & {$[0,33 ; 0,64]$} & $<0,001$ & 0,47 & {$[0,34 ; 0,65]$} & $<0,001$ \\
\hline \multicolumn{7}{|l|}{ Renda domiciliar mensal per capita } \\
\hline Sem renda ou até 1 salário mínimo & 0,53 & {$[0,49 ; 0,58]$} & $<0,001$ & 0,53 & {$[0,49 ; 0,58]$} & $<0,001$ \\
\hline Mais de 1 a 5 salários mínimos & 0,71 & {$[0,65 ; 0,77]$} & $<0,001$ & 0,71 & {$[0,65 ; 0,77]$} & $<0,001$ \\
\hline Mais de 5 salários mínimos & 1 & - & - & 1 & - & - \\
\hline Sem declaração & 0,69 & {$[0,61 ; 0,78]$} & 0,001 & 0,69 & {$[0,61 ; 0,78]$} & $<0,001$ \\
\hline \multicolumn{7}{|l|}{ Situação de ocupação } \\
\hline Ocupada & 1,19 & {$[1,15 ; 1,23]$} & $<0,001$ & 1,19 & {$[1,15 ; 1,23]$} & $<0,001$ \\
\hline Não ocupada & 1 & - & - & 1 & - & - \\
\hline \multicolumn{7}{|l|}{ Atividade física } \\
\hline Pratica & 1,36 & {$[1,31 ; 1,41]$} & $<0,001$ & 1,36 & {$[1,31 ; 1,41]$} & $<0,001$ \\
\hline Não pratica & 1 & - & - & 1 & - & - \\
\hline Sem declaração & 0,50 & {$[0,46 ; 0,55]$} & $<0,001$ & 0,50 & {$[0,46 ; 0,56]$} & $<0,001$ \\
\hline \multicolumn{7}{|l|}{ Fumo } \\
\hline Fumante & 0,86 & {$[0,82 ; 0,89]$} & $<0,001$ & 0,86 & {$[0,82 ; 0,89]$} & $<0,001$ \\
\hline Ex-fumante & 0,88 & {$[0,85 ; 0,91]$} & $<0,001$ & 0,88 & {$[0,85 ; 0,91]$} & $<0,001$ \\
\hline Nunca fumou & 1 & - & - & 1 & - & - \\
\hline Sem declaração & 0,95 & {$[0,92 ; 0,99]$} & 0,012 & 0,95 & {$[0,91 ; 0,99]$} & 0,009 \\
\hline \multicolumn{7}{|l|}{ Morbidade autorreferida } \\
\hline Pelo menos uma doença crônica & 0,29 & {$[0,28 ; 0,30]$} & $<0,001$ & 0,33 & {$[0,31 ; 0,36]$} & $<0,001$ \\
\hline Nenhuma doença crônica & 1 & - & - & 1 & - & - \\
\hline
\end{tabular}


Tabela 2. Modelos logísticos ordinais (multivariados) explicativos do estado de saúde autorreferido de adultos - Brasil.

\begin{tabular}{|c|c|c|c|c|c|c|}
\hline \multirow[t]{2}{*}{ Variáveis } & \multicolumn{3}{|c|}{$\begin{array}{l}\text { Modelo multivariado } 1 \text { : } \\
\text { inclui os efeitos principais } \\
\text { significativos }\end{array}$} & \multicolumn{3}{|c|}{$\begin{array}{l}\text { Modelo multivariado 2: } \\
\text { inclui os efeitos principais e } \\
\text { de interação significativos }\end{array}$} \\
\hline & $\begin{array}{l}\text { Razão de } \\
\text { chance }\end{array}$ & IC $95 \%$ & p-valor & $\begin{array}{l}\text { Razão de } \\
\text { chance }\end{array}$ & IC $95 \%$ & p-valor \\
\hline \multicolumn{7}{|l|}{ Mobilidade física } \\
\hline Muita limitação & 0,13 & {$[0,12 ; 0,14]$} & $<0,001$ & 0,13 & {$[0,12 ; 0,14]$} & $<0,001$ \\
\hline Limitação & 0,18 & {$[0,17 ; 0,19]$} & $<0,001$ & 0,18 & {$[0,17 ; 0,19]$} & $<0,001$ \\
\hline Pouca limitação & 0,42 & {$[0,40 ; 0,43]$} & $<0,001$ & 0,42 & {$[0,40 ; 0,43]$} & $<0,001$ \\
\hline Sem limitação & 1 & - & - & 1 & - & - \\
\hline \multicolumn{7}{|l|}{ Posse de plano de saúde } \\
\hline Sim & 1,32 & {$[1,26 ; 1,37]$} & $<0,001$ & 1,31 & {$[1,26 ; 1,37]$} & $<0,001$ \\
\hline Não & 1 & - & - & 1 & - & - \\
\hline \multicolumn{7}{|l|}{ Consulta médica } \\
\hline Sim & 0,55 & {$[0,53 ; 0,57]$} & $<0,001$ & 0,55 & {$[0,53 ; 0,58]$} & $<0,001$ \\
\hline Não & 1 & - & - & 1 & - & - \\
\hline \multicolumn{7}{|l|}{ Posse de bens básicos } \\
\hline Tem todos os quatro bens básicos & 1,14 & {$[1,10 ; 1,19]$} & $<0,001$ & 1,02 & {$[0,95 ; 1,10]$} & 0,540 \\
\hline Não tem pelo menos um bem básico & 1 & - & - & 1 & - & - \\
\hline$\%$ domicílios adequados & 1,36 & {$[1,26 ; 1,46]$} & $<0,001$ & 0,98 & {$[0,78 ; 1,23]$} & 0,864 \\
\hline \multicolumn{7}{|l|}{ Área`Sexo } \\
\hline Urbana * Masculino & & & & 0,86 & {$[0,81 ; 0,92]$} & $<0,001$ \\
\hline \multicolumn{7}{|l|}{ Área*Cor/raça } \\
\hline Urbana ${ }^{*}$ Branca & & & & 1,17 & {$[1,08 ; 1,27]$} & $<0,001$ \\
\hline \multicolumn{7}{|l|}{ Área* Morbidade autorreferida } \\
\hline Urbana * Pelo menos uma doença & & & & 0,83 & {$[0,76 ; 0,90]$} & $<0,001$ \\
\hline \multicolumn{7}{|l|}{ Área* Posse de bens básicos } \\
\hline Urbana $*$ Todos os bens & & & & 1,17 & {$[1,08 ; 1,28]$} & $<0,001$ \\
\hline \multicolumn{7}{|l|}{ Área* \% domicílios adequados } \\
\hline Urbana $* \%$ domicílios adequados & & & & 1,40 & {$[1,11 ; 1,77]$} & 0,005 \\
\hline
\end{tabular}

* Teste Wald. Nota: Excluídas as variáveis condição de ocupação do domicílio, qualidade da moradia e PSF no ajuste do modelo multivariado.

explicativo do estado de saúde autorreferido dos indivíduos, apenas as variáveis condição de ocupação do domicílio, qualidade da moradia e PSF foram excluídas do modelo por não apresentarem efeito estatisticamente significante ao nível de $5 \%$. Portanto, baseado no modelo multivariado 1, observa-se que, ao controlar pelas demais variáveis ambientais e individuais, a associação entre a área de localização do domicílio e o estado de saúde autorreferido dos adultos se modifica (passando de $\mathrm{OR}=1,51$ para $\mathrm{OR}=0,96)$ e perde a sua significância estatística ( $p$-valor=0,208).

Ao incluir todos os efeitos de interação das variáveis selecionadas no modelo multivariado 1 com a área de localização do domicílio, observou-se existência de interação para as seguintes variáveis: sexo, cor/raça, morbidade autorreferida, posse de bens básicos e o percentual de unidades adequadas, no setor de localização do domicílio (vizinhança), quanto à densidade, a infra-estrutura física e de serviços sociais (Tabela 2).

Devido à existência de interações no modelo multivariado 2 algumas medidas de associação não estão explicitadas na tabela 2 e precisam ser calculadas. Os homens na área urbana $\left(\mathrm{OR}=0,93^{\star} 0,96^{\star} 0,86=0,77\right)$ apresentam níveis de saúde menores que as mulheres na área urbana $\left(\mathrm{OR}=0,93^{\star} 1^{\star} 1=0,93\right)$, ou mesmo, que os homens na área rural $\left(\mathrm{OR}=1^{\star} 0,96^{\star} 1=0,96\right)$, indicando que em áreas urbanas o adulto do sexo masculino tem piores níveis de saúde. Já os brancos apresentam níveis de saúde maiores do que os não brancos, mas na área urbana $\left(\mathrm{OR}=0,93^{*} 1,02^{*}\right.$ $1,17=1,11)$ estes níveis são ligeiramente maiores que na área rural $\left(\mathrm{OR}=1^{\star} 1,02^{\star} 1=1,02\right)$.

Os adultos que residem em domicílios que possuem todos os bens básicos têm níveis de saúde maiores do que aqueles que residem em do- 
micílios sem pelo menos um dos bens básicos. Entretanto, é na área urbana que os níveis de saúde dos adultos residentes em domicílios com todos os bens são ligeiramente maiores (urbana: $\mathrm{OR}=0,93^{\star} 1,02^{\star} 1,17=1,11$ e rural: $\mathrm{OR}=1^{\star} 1,02^{\star}$ $1=1,02)$. Com relação aos adultos com pelo menos uma doença crônica, observam-se menores níveis de saúde na área urbana $\left(\mathrm{OR}=0,93^{*}\right.$ $\left.0,33^{\star} 0,83=0,25\right)$ do que na área rural $\left(\mathrm{OR}=1^{\star}\right.$ $0,33^{\star} 1=0,33$ ).

Embora a variável qualidade da moradia (ambiente intradomiciliar) não tenha apresentado significância estatística no ajuste do modelo multivariado, o percentual de unidades adequadas quanto à qualidade da moradia no setor de localização do domicílio (ambiente extradomiciliar) está fortemente associado com uma autopercepção positiva do estado de saúde na área urbana $\left(\mathrm{OR}=0,93^{\star} 0,98^{\star} 1,40=1,28\right) . \mathrm{Na}$ área rural, este efeito não foi significativo.

Entre os resultados do modelo multivariado 2 cabe destacar ainda que as variáveis faixa-etária, escolaridade, renda domiciliar mensal per capita e mobilidade física mantêm os gradientes com a chance de autopercepção do estado de saúde de adultos, mas não as magnitudes dos efeitos ( $\mathrm{Ta}$ bela 2), quando comparados com os resultados dos modelos univariados (Tabela 1 ).

\section{Discussão}

Para a análise das diferenças no estado de saúde autorreferido dos indivíduos, os estudos eram mais frequentemente conduzidos no campo da epidemiologia sob a ótica reducionista levando em conta características individuais, em detrimento de características do ambiente ${ }^{19}$; não predominando, portanto a chamada visão neomaterial ${ }^{4,20}$. O presente trabalho, considera um indicador de auto percepção de saúde como proxy da avaliações objetivas de saúde ${ }^{8}$, e os resultados da análise estatística efetuadas evidenciam a existência de associação entre este indicador e fatores do ambiente intradomiciliar e extradomiciliar, além de fatores individuais. Apesar de sua natureza subjetiva, o estado de saúde autorreferido tem sido apontado na literatura como um preditor de alta validade da mortalidade, independentemente de fatores de riscos médicos, comportamentais ou psicossociais ${ }^{21}$. Além de sua validade, vários estudos têm demonstrado que a autopercepção de saúde é uma medida que apresenta boa confiabilidade $^{8-10}$. Além disso, segundo Nogueira ${ }^{4}$, a subjetividade não é uma limitação e sim um pon- to forte deste indicador, tendo em vista que o processo saúde-doença é um processo contínuo, relativo e subjetivo, cuja percepção, cognição e interpretação variam no tempo e no espaço, em função de fatores individuais e contextuais.

Independentemente da área de localização do domicílio, observou-se que os indivíduos mais escolarizados apresentam melhores níveis de estado global de saúde, assim como os indivíduos residentes em domicílios com maiores rendimentos per capita. As associações das variáveis escolaridade e rendimento per capita com a autopercepção de saúde (dicotomizada) também foram identificadas por Dachs e Santos ${ }^{13}$ e Kassouf ${ }^{22}$. A importância da última variável na explicação das diferenças no estado de saúde autorreferido devese ao fato do rendimento, condicionando os indivíduos nas suas opções quotidianas - impondo ou não, limitações à quantidade e à qualidade de bens possíveis de adquirir, e restringindo, ou não, a mobilidade e a vida social - afeta a saúde física $e$ mental ${ }^{4}$.

A ocupação, considerada como um dos principais meios de acesso à renda e aos serviços sociais básicos ${ }^{22}$, também está fortemente associada a melhores níveis de saúde. A exclusão do indivíduo do mercado de trabalho, além de gerar privação material, pode levá-lo a restrição de direitos, de segurança socioeconômica e de autoestima $^{23}$

Considerando a região Centro-Oeste como referência, observa-se que a região Sudeste é a região brasileira que concentra indivíduos que reportam melhores níveis de saúde. Os indivíduos das regiões Norte e Nordeste reportaram piores estados de saúde do que os da região Centrooeste. Já a região Sul não apresentou efeito estatisticamente significante. Kassouf ${ }^{22}$ também observou que, além da Região Sul, indivíduos da região Sudeste apresentam um melhor estado de saúde do que os da região Nordeste, seguido da região Norte.

Independente da área ser urbana ou rural, observou-se o efeito prejudicial do fumo na saúde dos indivíduos, indicando que tanto os fumantes quanto os ex-fumantes avaliam piores estados de saúde, comparativamente aos indivíduos que nunca fumaram. Por outro lado, observa-se o efeito benéfico da prática de atividade física no estado geral de saúde, como também verificado no estudo de Höfelmann e Blank ${ }^{14}$.

Indivíduos mais velhos reportaram piores níveis de estado de saúde, assim como os indivíduos que reportaram maior grau de dificuldade na realização de determinadas atividades do co- 
tidiano. A mobilidade física prejudicada, definida como estado no qual a pessoa sofre uma limitação no movimento físico independente e voluntário do corpo ou de uma ou mais extremidades $^{24}$, é um dos problemas que mais compromete a saúde das pessoas, sendo os idosos considerados o grupo mais suscetível a desenvolvê-la, uma vez que durante o processo de envelhecimento os idosos sofrem modificações importantes na capacidade motora ${ }^{25}$.

Quanto à consulta médica, observou-se também, independentemente da área de localização do domicílio, uma relação negativa com a autoavaliação do estado de saúde, indicando que adultos que consultaram médico nos últimos doze meses avaliam um pior estado de saúde do que aqueles que não consultaram neste período. Tal resultado parece estar condizente com a conclusão obtida por Kassouf ${ }^{22}$ de que indivíduos com melhores níveis de saúde parecem reduzir a procura por atendimento à saúde.

Ainda em relação às características de saúde, conclui-se que indivíduos que possuem plano de saúde, médico ou odontológico, particular, de empresa ou órgão público apresentam melhores níveis de estado de saúde do que os indivíduos sem um. Esse resultado parece estar relacionado ao fato de que indivíduos portadores de plano de saúde possuem um maior acesso e utilização a serviços de saúde ${ }^{22}$.

Com relação à modelagem estatística, é importante ressaltar que foram considerados os pesos amostrais e as informações estruturais do plano amostral da PNAD 2008. Quando se ignora estas informações, a análise efetuada a partir de dados de amostras complexas pode produzir resultados incorretos tanto para as estimativas pontuais como para as medidas de precisão e pvalores, o que, por sua vez, pode comprometer o ajuste de modelos e a interpretação dos resultados obtidos ${ }^{15,17,26,27}$.

Outro aspecto que merece destaque é a utilização do modelo logístico ordinal ${ }^{18,28}$, que considerou a natureza policotômica ordinal do desfecho de saúde utilizado, sem dicotomizá-lo. Além disso, considerou fatores individuais e ambientais (intradomiciliar e extradomiciliar) para explicar as diferenças nos níveis de autopercepção de saúde, embora não se tenha considerado a estrutura hierárquica dos dados, por meio de modelagem multinível ${ }^{29}$. Através da análise multinível seriam incorporados explicitamente os múltiplos níveis de informação (indivíduo, domicílio, setor) no modelo, possibilitando explicar a contribuição de cada um na variação do desfecho estudado. Entretanto, isso introduziria uma complexidade ainda maior na análise devido à necessidade de se incorporar ainda os diferentes aspectos do plano amostral da PNAD (estratos, unidades primárias de amostragem e pesos amostrais $)^{26}$.

No que se refere à qualidade do ajuste do modelo multivariado 2 (Tabela 2), observa-se que $42,0 \%$ da variação dos níveis de estado de saúde autorreferido (variável latente) é explicada pelo modelo ajustado. Além do valor moderado para a medida de qualidade do ajuste (pseudo $\mathrm{R}^{2}$ de Nagelkerke), observa-se também que o modelo prediz, com diferentes graus de acurácia, os três níveis de estado de saúde considerados, sendo que a porcentagem de previsões corretas é de 90,1\% para o nível "bom/muito bom"; 50,3\% para o nível "regular" e de 6,6\% para o nível "ruim/muito ruim". Em termos globais, o modelo selecionado classifica corretamente $75,1 \%$ dos adultos.

Uma das limitações deste estudo pode estar na própria definição político-administrativa de urbano-rural baseada em leis municipais como é no caso do Brasil ${ }^{30}$. Segundo Endlich ${ }^{31}$ a falta de uma definição oficial mais criteriosa do que é cidade no Brasil provoca grandes discussões a respeito de como distinguir os limites entre urbano e rural. Há críticas quando a definição desses limites baseada apenas no conceito político-administrativo utilizado pelo IBGE, não considerando assim outros critérios ${ }^{31,32}$. Devido à complexidade relacionada ao tema, vários critérios são apontados na literatura para definição de cidade, entre os quais pode-se destacar o tamanho geográfico, densidade, aspectos formológicos, atividades econômicas e modo de vida. Segundo Bernardelli ${ }^{32}$ os critérios usados para o processo emancipatório dos municípios no passado, e ainda presente hoje, estão muitas vezes meramente associados a interesses políticos e tributários, servindo para beneficiamento de alguns grupos e partidos, bem como para atingir suas demandas. Esta autora acrescenta ainda, que muitas vezes municípios sem receitas próprias são emancipados, acarretando dificuldades administrativas e não beneficiamento da população residente neles.

Outra limitação deste estudo se refere à impossibilidade de se considerar outras variáveis importantes, tais como as de natureza nutricional ${ }^{33}$ e de poluição atmosférica ${ }^{34}$, por não fazerem parte do escopo do suplemento da saúde da PNAD 2008.

Conclui-se que a área urbana parece influenciar negativamente o estado de saúde autorreferido, uma vez que nestas áreas adultos com de- 
terminadas características (sexo masculino; raça não branca; portador de pelo menos uma doença crônica; residente em domicílios sem pelo menos um dos bens básicos; e residente em setores com menores percentuais de domicílios adequados quanto à qualidade da moradia) reportam piores níveis de estado de saúde.

\section{Colaboradores}

JR Moraes, JPL Moreira e RR Luiz participaram igualmente de todas as etapas da elaboração do artigo.

\section{Agradecimentos}

Este artigo foi parcialmente financiado com recursos da FAPERJ.

\section{Referências}

1. Caiaffa WT, Ferreira FR, Ferreira AD, Oliveira CDL, Camargos VP, Proietti FA. Saúde urbana: "a cidade e uma estranha senhora, que hoje sorri e amanhã te devora". Cienc Saude Colet 2008;13(6): 1785-1786.

2. Instituto Brasileiro de Geografia e Estatística (IBGE). Pesquisa Nacional por Amostra de Domicílios 2008 Notas Metodológicas: pesquisa básica, pesquisa especial de tabagismo e pesquisas suplementares de saúde e acesso à internet e posse de telefone móvel celular para uso pessoal. Rio de Janeiro: IBGE; 2010. [acessado 2010 abr]. Disponível em: http://www.ibge. gov.br

3. Mcdade T, Adair L. Defining the Urban in Urbanization and Health: A Factor Analysis Approach. Soc Sci Med 2001; 53:55-70.

4. Nogueira H. Os Lugares e a Saúde. Coimbra: Imprensa da Universidade de Coimbra; 2008.

5. Vlahov D, Galea S, Freudenberg N. Perspectives on urban conditions and population health. Cad Saude Publica 2005; 21(3):949-957.

6. Moore M, Gould P, Keary B. Global urbanization and impact on health. International Journal of Environmental Health 2003; 203:269-278.

7. Azeredo C M, Cotta RMM, Schott M, Maia TM, Marques ES. Avaliação das condições de habitação e saneamento: a importância da visita domiciliar no contexto do Programa de Saúde da Família. Cienc Saude Colet 2007; 12(3):743-753.

8. Camargos MCS, Rodrigues RN, Machado CJ. Expectativa de vida saudável para idosos brasileiros, 2003. Cienc Saude Colet 2009; 14(5):1903-1909.

9. Martikainen P, Aromaa A, Heliövaara M, Klaukka T, Knekt P, Maatela J, Lahelma E. Reliability of perceived health by sex and age. Soc Sci Med 1999; 48(8):1117-1122.

10. Bailis DS, Segall A, Chipperfield JG. Two views of selfrated general health status. Soc Sci Med 2003; 56(2):203-217.

11. Souza EM; Abrão FPS; Motta IA, Almeida JO. Autopercepção do estado de saúde: um estudo de prevalência com adolescentes de Ceilândia, Distrito Federal, Brasil. Comun Cienc Saúde 2006; 17(1):9-15.

12. Barros MBA; Zanchetta LM, Moura EC, Malta DC. Auto-avaliação da saúde e fatores associados, Brasil, 2006. Rev Saude Publica 2009; 43(2):27-37.

13. Dachs JNW, Santos APR. Auto-avaliação do estado de saúde no Brasil: análise dos dados da PNAD/ 2003. Cien Saude Colet 2006; 11(4):887-894. 
14. Hofelmann DA, Blank N. Auto-avaliação de saúde entre trabalhadores de uma indústria no sul do Brasil. Rev Saude Publica 2007; 41(5):777-787.

15. Silva PLDN, Pessoa DGC, Lila MF. Análise Estatística de Dados da PNAD: Incorporando a Estrutura do Plano Amostral. Cienc Saude Colet 2002; 7(4):659670 .

16. Instituto Brasileiro de Geografia e Estatística (IBGE). Pesquisa sobre padrões de vida 1996-1997. Rio de Janeiro: DPIS, IBGE; 1999.

17. Lee ES, Forthofer RN, Lorimor RJ. Analyzing complex survey data. Newbury Park: Sage Publications; 1989.

18. Abreu MNS, Siqueira AL, Caiaffa WT. Regressão logística ordinal em estudos epidemiológicos. Rev Saude Publica 2009; 43(1):183-94.

19. Proietti FA, Oliveira CDL, Ferreira FR, Ferreira AD, Caiaffa WT. Unidade de Contexto e Observação Social Sistemática em Saúde: Conceitos e Métodos. Physis 2008; 18(3):469-482.

20. Townsend P, Davidson N. The Back Report. In: Townsend P, Davidson N (Editors.). Inequalities in Health. Suffolk: Penguin Books Ltd.; 1988.

21. Idler EL, Benyamini Y. Self-rated health and mortality: a review of twenty-seven community studies. J Health Social Behav 1997; 38(1):21-37.

22. Kassouf, AL. Acesso aos serviços de saúde nas áreas urbana e rural do Brasil. Rev Econ Sociol Rural 2005; 43(1):29-44.

23. Pochmann M. O trabalho sob fogo cruzado: exclusão, desemprego e precarização no final do século. São Paulo: Contexto; 1999.

24. North American Nursing Diagnosis Association (NANDA), Michel JLM, tradutora. Diagnósticos de Enfermagem da NANDA. Definições e classificação 1999-2000. Porto Alegre: Artmed; 2000.

25. Bachion M.M, Araújo LAO, Santana RF. Validação de conteúdo do diagnóstico de enfermagem "Mobilidade Física Prejudicada" em idosos: uma contribuição. Acta Paul Enf 2002; 15(4):66-72.

26. Pessoa DGC, Silva PL. Análise de dados amostrais complexos. São Paulo: ABE; 1998.
27. Szwarcwald CL, Damacena GN. Amostras complexas em inquéritos populacionais: planejamento e implicações na análise estatística dos dados. Rev Bras Epidemiol 2008; 11(Supl. 1):38-45.

28. Maroco J. Análise Estatística com o PASW Statistics (ex-SPSS). Pêro Pinheiro: ReportNumber; 2010.

29. Diez-roux AV. Bringing context back into epidemiology: Variables and fallacies in multilevel Analysis. Am J Public Health 1998; 88(2):216.

30. Camello TCF, Garcia VS, Araújo SB, Almeida JR. Gestão e vigilância em saúde ambiental. Rio de Janeiro: Thex; 2009.

31. Endlich AM. Perspectivas sobre o urbano e o rural. In: Sposito MEB, Whitacker AM, organizadores. Cidade e Campo: relações e contradições entre urbano e rural. $1^{\text {a }}$ ed. São Paulo: Expressão Popular; 2006.

32. Bernardelli MLFH. Contribuição ao debate sobre o urbano e o rural. In: Sposito MEB, Whitacker AM, organizadores. Cidade e Campo: relações e contradições entre urbano e rural. $1^{\text {a }}$ ed. São Paulo: Expressão Popular; 2006.

33. Cuppari L. Guia de Nutrição: Nutrição Clínica no Adulto. $1^{a}$ ed. São Paulo: Manole; 2002. (Coleção Guia de Medicina Ambulatorial e Hospitalar UNIFESP, Escola Paulista de Medicina).

34. Esteves GRT, Seixasbarbosa SRC, Silva EP, Duarte P. Estimativas do Efeito da Poluição Atmosférica sobre a Saúde Humana: algumas possibilidades metodológicas e teóricas para cidade de São Paulo. In: Encontro da Associação Nacional de Pós-Graduação e Pesquisa em Ambiente e Sociedade; 2004; São Paulo. p.1-20.

Artigo apresentado em 21/10/2010

Aprovado em 16/02/2011

Versão Final apresentada em 25/02/2011 